\title{
Temperature-Dependence of Electrical Conductivity for Some Natural Coordination Polymeric Biomaterials Especially Some Cross-Linked Trivalent Metal-Alginate Complexes with Correlation between the Coordination Geometry and Complex Stability
}

\section{Ishaq A Zaafarany*}

Chemistry Department, Faculty of Applied Sciences, Umm Al-Qura University, Makkah Al-Mukarramah 13401, Saudi Arabia

\begin{abstract}
The electrical conductivity (o') of cross-linked arsenic (III) - alginate complex as a coordination polymeric biomaterial in the form of circular discs have been measured as a function of temperature. The measured values of the electrical conductivity were found to be in the range of semiconductors. The change of electrical conductivity as a function of temperature was found to be of considerable complexity (Arrhenius plot of In o' vs. $1 / T$ ). The appearance of a parabola zone at the early stages was explained by the release of waters of crystalline, whereas the sharp increase in o values observed at the elevated temperatures was interpreted by the degradation process of the complex to give rise to the metal oxide as final product. The X-ray diffraction patterns indicated that the metalalginate complex is amorphous in nature. Infrared absorption spectra revealed a sort of complexation between the trivalent metal cations and the functional carboxylate and hydroxyl groups of alginate macromolecule. A suitable conduction mechanism in terms of the complex stability in relation to the coordination geometry is suggested and discussed.
\end{abstract}

Keywords: Polymeric biomaterials; Alginate, Arsenic (III); Complexes; Semiconductors; Electrical conductivity.

\section{Introduction}

Alginate is a binary linear heteropolymer consisting of 1,4- linked $\beta$-D-mannuronic and $\alpha$-L- guluronic acids units. The monomers are arranged in clockwise fashion along the macromolecular chains [1].

Conducting polymers offer the promise of achieving a new generation of polymers and materials that exhibit a high potential application in industrial technology [2-6]. Conductance measurements of polymers that sandwiched between two metal electrodes have attracted much attention owing to its potential applications in electronic devices [7] as well as to gain more information on the structure of materials that may be achieved from such measurements.

Although, conducting polymers in particularly that prepared from synthetic polymeric materials have been the subject of continuous research and development in recent years owing to their unique electrical, optical and chemical properties [8], a little attention has been focused to that of natural polymers such as the coordination polymeric biomaterials such as cross-linked metal-alginate complexes. This fact may be attributed to a lack of information on the electrical properties of such natural polysaccharide complexes. Even though, Hassan and co-workers studied the behavior of electrical conductivity for some coordination biopolymeric polysaccharides such as cross-linked metal-alginate complexes. For example the electrical conductivity of such complexes in either granule $[8,9]$ or the gel forms $[10,11]$ was investigated as a function of frequency earlier. Again, the electrical conductivity as a function of temperature for some metal-alginate complexes such as monovalent [12], divalent [13], trivalent [14,15], tetravalent [16-18] and hexavalent [19] metal cations in the granule forms have been studied elsewhere. Unfortunately, the suggested conduction mechanisms in these complexes are still incomplete owing to the discrepancies and variety observed in the behavior of electrical conductivity of studied complexes. It was found that no change in the electrical conductance with raising temperature for complexes involving chelated divalent [13] and trivalent [14] metal ions of one- equivalent nature and the values of the electrical conductivity obtained (o) lies in the range of insulators. On the other hand, metal alginate complexes involving trivalent [15], tetravalent [16-18] and hexavalent [19] metal ions of multi-equivalent nature showed an increase in the electrical conductivity with raising the temperature through parabolic shapes depending on the possible number of oxidation states formed during the reduction of chelated metal ion in that complex.

Preliminary experiments of the cited As (III)-alginate complex showed complicated behavior with respect to the trend of electrical conductance change with raising the temperature. However, As (III) ion cannot be reduced to another form, a parabola zone was appeared at the early stages, whereas a sharp increase in ( $\circlearrowleft$ ) values followed by a sharp decrease in these values at higher temperatures was observed on contrary to that observed previously in similar complexes $[13,14]$.

In view of the above discrepancies and our interest in physicochemial studies of such natural coordination biopolymeric complexes, the electrical conductivity of the cited complex seems to be of great significant and merits an investigation with the aims of shedding some light on the conduction mechanism in these metal-alginate complexes as well as to gain further information on the correlation between the electrical properties, geometrical structure and the stability in these

*Corresponding author: Ishaq A Zaafarany, Chemistry Department, Faculty of Applied Sciences, Umm Al-Qura University, Makkah Al-Mukarramah 13401, Saudi Arabia, Tel: +966 503599574; E-mail: ishaq_zaafarany@yahoo.com

Received: January 10, 2014; Accepted: August 4, 2014; Published: August 11, 2014

Citation: Zaafarany IA (2014) Temperature-Dependence of Electrical Conductivity for Some Natural Coordination Polymeric Biomaterials Especially Some CrossLinked Trivalent Metal-Alginate Complexes with Correlation between the Coordination Geometry and Complex Stability. J Adv Chem Eng 4: 111. doi: 10.4172/2090-4568.1000111

Copyright: (c) 2014 Zaafarany IA. This is an open-access article distributed under the terms of the Creative Commons Attribution License, which permits unrestricted use, distribution, and reproduction in any medium, provided the original author and source are credited. 
Citation: Zaafarany IA (2014) Temperature-Dependence of Electrical Conductivity for Some Natural Coordination Polymeric Biomaterials Especially Some Cross-Linked Trivalent Metal-Alginate Complexes with Correlation between the Coordination Geometry and Complex Stability. J Adv Chem Eng 4: 111. doi: 10.4172/2090-4568.1000111

Page 2 of 6

coordination polymeric biomaterial complexes as well as to compare the results obtained with those reported previously for other metalalginate complexes.

\section{Materials and Methods}

\section{Reagents}

Sodium alginate used was Cica-Reagent (Kanto Chem. Co.). Doubly distilled conductively water was used in all preparations. All other materials used were of analytical grade.

All other materials were of BDH grade (with purity 99.99\%) and were used without further purification. Doubly-distilled water was used in all preparations.

\section{Preparation of As (III)-alginate granule complex}

Generally, cross-linked metal-alginate granule complexes are prepared by the replacement of $\mathrm{Na}^{+}$counter ions in alginate macromolecule by the corresponding polyvalent metal cations as described elsewhere [12-19]. This process was performed by stepwise addition of the alginate powder to the electrolyte solution of the As (III) ions while rapidly stirring the solution to avoid the formation of lumps, which swells with difficulty. After completion of the exchange process, the grains formed were washed with deionized water until the resultant water became free from non-chelated metal ions and then dried under vacuum over anhydrous $\mathrm{CaCl}_{2}$ or $\mathrm{P}_{2} \mathrm{O}_{5}$.

Samples in the form of circular discs with diameter $13 \mathrm{~mm}$ and thickness 1-2 mm were obtained using an IR disc press at a constant pressure of 1500 psi.

\section{$\mathrm{X}$-ray diffraction}

The X-ray diffraction patterns were obtained using a Philip 1710 diffractometer as described elsewhere [12-19].

\section{IR spectra}

The IR spectra were scanned on a Pye Unicam Sp 3100 spectrophotometer using the $\mathrm{KBr}$ disc technique $\left(4000-400 \mathrm{~cm}^{-1}\right)$.

\section{Conductance measurements}

The specific mechanical properties of metal-alginate complexes in gel form make the measurements of their electrical properties by the usual methods very difficult. Therefore, a new technique of measurements involving a new cell device has been devised by us in order to overcome such difficulty $[10,11]$

The AC conductance was measured over the temperature range 290-560 K using a Keithely 610 C electrometer. The electrodes used were of circular shape forming M-S-M sandwich, where $M$ represents the electrodes and $\mathrm{S}$ is the specimen. The metal-alginate complex discs were sandwiched between the two standard electrodes (graphite, copper or silver paste) mounted into a specially designed temperaturecontrolled electric furnace provided with a standard copper-constantan thermocouple. The samples were thermostated by kept short circuited for $3-5 \mathrm{~h}$ in air-thermostat within $\pm 1.0^{\circ} \mathrm{C}$ to make it ready for experiments. The electrical resistance of the samples was measured, and from this the electrical conductivities ( $\sigma$ ) were calculated as follows

$$
\sigma=(1 / \mathrm{R})(\mathrm{L} / \mathrm{a})
$$

where R is the resistance $(\Omega)$, a is the area of the sample surface $\left(\mathrm{cm}^{2}\right)$ and $\mathrm{L}$ is the thickness of the specimen $(\mathrm{cm})$.

\section{Results and Discussion}

Cross-linked trivalent metal-alginate complexes are formed when the $\mathrm{Na}^{+}$counter ions of alginate macromolecule are replaced by an equivalent amount of quadrivalent metal cations. The interdiffused metal cations chelate the carboxylate and hydroxyl functional groups of alginate macromolecular chains through formation of partially ionic and partially coordinate bonds [20], respectively, and mediated through blocks of alginate macromolecular chains in an egg-carton like structure [20-23]. This exchange process occurs inherently and stoichiometrically even the valences and mobilities of the two exchanging counter ions are quite different [24].

$$
\begin{aligned}
& 3(\mathrm{Alg}-\mathrm{Na})_{\mathrm{n}}+\mathrm{n} \mathrm{M}^{3+} \rightarrow\left(\mathrm{Alg}_{3}-\mathrm{M}\right)_{\mathrm{n}}+3\left(\mathrm{Na}^{+}\right)_{\mathrm{n}} \\
& \text { solid aqueous solid complex aqueous }
\end{aligned}
$$

where $\left.(\mathrm{Alg}-\mathrm{Na}), \mathrm{Alg}_{3}-\mathrm{M}\right)$ and $\mathrm{M}$ are sodium alginate, trivalent metal alginate complex and the interdiffused metal ion (As(III)), respectively.

The X-ray diffraction patterns indicate that the investigated alginate complex is amorphous in nature. Relevant infrared bands which provide considerable structural evidence for the mode of attachment between these trivalent metal cations and alginate functional groups are shown in Figure 1. The vibrational assignments of the bands showed that the bands of $v_{\mathrm{s}} \mathrm{CO}_{2}{ }^{-}$and $v_{2 \mathrm{~s}} \mathrm{CO}_{2}{ }^{-}$are shifted from 1600 and $1400 \mathrm{~cm}^{-1}$ in alginate to higher frequencies in the complexes, indicating the complexation between the interdiffused metal cations and the carboxylate and hydroxyl functional groups of alginate macromolecular chains $[25,26]$.

The broad band observed at around $3500 \mathrm{~cm}^{-1}$ which belongs to $v_{\mathrm{OH}}$ of water (or $\mathrm{OH}$-free functional groups) is also shifted to lower frequencies as shown in Table 1. The free-ligand has a strong band at $1735 \mathrm{~cm}^{-1}$ which can be assigned to the carbonyl stretching vibration of the carbonyl group [25]. The displacement of this band at around $1742-1747 \mathrm{~cm}^{-1}$ may also indicate the coordination of the carboxylate groups with the appearance of both symmetric $\left(v_{\mathrm{s}}\right)$ and asymmetric $\left(v_{\mathrm{as}}\right)$ vibrations of $\mathrm{COO}^{-}$group. Again, the location of $v_{\mathrm{s}} \mathrm{COO}^{-}$is a diagnostic of the bridging carboxylate groups.

The electrical conductivity of As (III)- alginate complex in the form

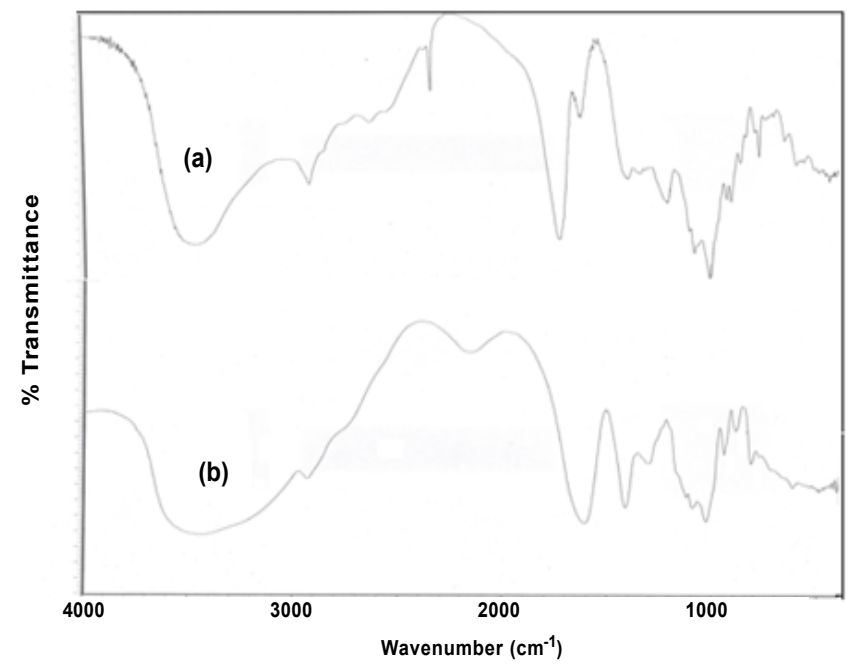

Figure 1: Infrared spectra of (a): As (III)-alginate complex; (b) alginate substrate. 
Citation: Zaafarany IA (2014) Temperature-Dependence of Electrical Conductivity for Some Natural Coordination Polymeric Biomaterials Especially Some Cross-Linked Trivalent Metal-Alginate Complexes with Correlation between the Coordination Geometry and Complex Stability. J Adv Chem Eng 4: 111. doi: 10.4172/2090-4568.1000111

\begin{tabular}{|c|c|c|c|c|c|}
\hline Complex & $\mathrm{u}_{\mathrm{s}} \mathrm{OCO}$ & $\mathrm{u}_{\mathrm{as}} \mathrm{OCO}$ & $\mathrm{u}_{\mathrm{OH}}$ & $\mathbf{U}_{\mathrm{M}-\mathrm{O}}$ & Reference \\
\hline Na-alginate & 1400 & 1600 & 3500 & 850 & 24,30 \\
\hline Ag'-alginate & 1400 & 1615 & 3444 & 830 & 12 \\
\hline As'I'-alginate & 1408 & 1634 & 3467 & 818 & This work \\
\hline La'"I-alginate & 1419 & 1598 & 3426 & 820 & 14 \\
\hline$C e^{\text {III-alginate }}$ & 1421 & 1618 & 3441 & 815 & 14 \\
\hline $\mathrm{Cr}^{\prime \prime \prime}$ - alginate & 1420 & 1637 & 3463 & 810 & 15 \\
\hline $\mathrm{Fe}^{\mathrm{III}}$-alginate & 1418 & 1633 & 3448 & 817 & 15 \\
\hline $\mathrm{Sn}^{\mathrm{IV}}$-alginate & 1414 & 1634 & 3457 & 811 & 16 \\
\hline $\mathrm{Zr}^{\prime \mathrm{IV}}$-alginate & 1408 & 1629 & 3447 & 813 & 16 \\
\hline $\mathrm{Se}^{\mathrm{IV}}$-alginate & 1408 & 1629 & 3387 & 806 & 17 \\
\hline $\mathrm{Th}^{\mathrm{IV}}$-alginate & 1419 & 1635 & 3461 & 890 & 18 \\
\hline $\mathrm{U}^{\mathrm{VI}}$-alginate & 1410 & 1591 & 3410 & 817 & 19 \\
\hline $\mathrm{Ce}^{\mathrm{IV}}$-alginate & 1424 & 1603 & 3445 & 809 & 37 \\
\hline
\end{tabular}

Notes: $U_{s}$ Symmetry stretching vibrations of OCO, $U_{s}$ : Assymetry stretching vibrations of $\mathrm{OCO}, \mathrm{U}_{\mathrm{OH}}$ : Stretching vibrations of $\mathrm{H}$ bond of $\mathrm{OH}, \mathrm{U}_{\mathrm{M}-\mathrm{O}}$ : Stretching vibrations of metal-oxygen bond.

Table 1: FTIR frequencies $\left(\mathrm{cm}^{-1}\right)$ for Sodium Alginate and Arsenic (III) cross linked Metal-Alginate complexes.

of circular disc was measured as a function of temperature. As is shown in Figure 2, a plot of ln ơ against 1/T of Arrhenius equation displayed a complicated behavior where a small parabola zone was appeared at the early stages, but a sharp increase in (o) values followed by a sharp decrease in these values was observed at the higher temperatures.

The formation of such parabolic zones was observed in analogous studies on the temperature-dependence of electrical conductivity for some other cross-linked metal-alginate complexes of multi-equivalent nature [15-19]. In those complexes, the observed parabolic behavior for the increase of the electrical conductivity with increasing the temperature at the early stages was explained by the formation of freeradical intermediates as a result of reduction of the chelated multiequivalent metal cations to lower oxidation states. This suggestion was based on the possibility of transfer of electrons from the alginate macromolecule to the cross-linked metal cations. The number of transition zones is mainly dependent on the possible oxidation states obtained from the reduction of metal ion. For example, chromium (III) and iron (III) are well-known to be of two-equivalent oxidant nature [27], i.e. $\mathrm{M}^{3+} \rightarrow \mathrm{M}^{2+}$ on reduction. Therefore, the number of expected transition zones should be only one as was experimentally observed [15]. Again, uranium (VI) is a multi-equivalent oxidant [28] which gives various oxidation states on reduction, $\mathrm{U}^{\mathrm{V}}, \mathrm{U}^{\mathrm{IV}}$ and $\mathrm{U}^{\mathrm{III}}$, through successive one-electron-transfer mechanism in a sequence by. So, three parabolic zones should be existed as was reported earlier [19]. On the other hand, the reduction of divalent- and some trivalent-metal cations of one-equivalent nature such as lanthanum (III) and cerium (III) cannot be reduced to lower oxidation states [27]. Hence, no parabolic zones should be expected as was confirmed experimentally $[13,14]$.

In view of the above interpretations and the experimental observations, the formation of small parabolic zone at the early stage with respect the behavior of electrical conductivity with increasing temperature (Figure 2) in As(III)-alginate complex cannot be attributed to reduction of chelated As(III) metal ion since no further lower oxidation states are known for arsenic ion are known [27]. The most probable interpretation of such observed parabola may be explained by the evolution of water of crystallization as was reported for analogous cases [12].

$$
\left[\left(\mathrm{RCOO}^{-}\right)_{3} \cdot \mathrm{As}^{\mathrm{III}}\right]_{\mathrm{n}} \rightarrow\left[\left(\mathrm{RCOO}^{-}\right)_{3(-\mathrm{xH} 2 \mathrm{O})} \cdot \mathrm{As}^{\mathrm{III}}\right]_{\mathrm{n} 1}+\mathrm{XH}_{2} \mathrm{O}
$$

where $\mathrm{RCOO}^{-}$represents to the alginate monomers $\left(\mathrm{C}_{6} \mathrm{H}_{7} \mathrm{O}_{6}^{-}\right)_{n}$

Again, the sharp increase in (o) values observed at higher temperatures over $200^{\circ} \mathrm{C}$ (I) can be explained by the degradation of the complex to form its corresponding arsenic carbonate radical [29] with evolution of carbon dioxide and water vapor as follows

$$
\left[\left(\mathrm{RCOO}^{-}\right)_{3(-\mathrm{xH} 2 \mathrm{O})} \cdot \mathrm{As}^{\mathrm{III}}\right]_{\mathrm{n} 1}+\alpha \mathrm{O}_{2} \rightarrow \mathrm{As}(\mathrm{OH})_{2}\left(\mathrm{CO}_{3}\right)+\mathrm{YCO}_{2}+\mathrm{ZH}_{2} \mathrm{O}
$$

Of course, the dimerization process of such free-radicals formed will lead to a decrease in the charge carriers and, hence, a decrease in the electrical conductivity should be expected as was experimentally observed (II). This dimerization can be expressed by the following equations.

$$
2\left(\mathrm{As}(\mathrm{OH})_{2}\left(\mathrm{CO}_{3}\right) \cdot\right) \rightarrow\left[\mathrm{As}(\mathrm{OH})_{2}\left(\mathrm{CO}_{3}\right)\right]_{2}
$$

On other hand, the sharp increase in ơ values observed at elevated temperatures (III) can be explained by the decomposition of the arsenic carbonate to give rise to its corresponding more stable metal oxide as final degradation products $[14,15,30,31]$

$$
\left[\mathrm{As}(\mathrm{OH})_{2}\left(\mathrm{CO}_{3}\right)\right]_{2}+1 / 2 \mathrm{O}_{2} \rightarrow \mathrm{As}_{2} \mathrm{O}_{5}+2 \mathrm{H}_{2} \mathrm{O}+2 \mathrm{CO}_{2}
$$

The values of electrical conductivities obtained using different electrodes were found to be in good agreement with each other confirming the reproducibility of the conductance measurements (within experimental errors of $\pm 3 \%$ ). The correlation coefficient of the data obtained was $\left(r^{2}>0.99\right)$. In other words, it means that the nature of electrodes has no influence on the behavior of electrical conductivity.

On the other hand, the kinetics of non-isothermal decomposition of some coordination biopolymer trivalent-metal alginate cross-linked complexes such as $\mathrm{Al}$ (III), $\mathrm{Cr}$ (III), $\mathrm{Fe}$ (III), Ce (III) and La (III) has been investigated using a Mettler TA 3000 thermal analyzer in static air and were reported elsewhere $[32,33]$. The TG curves showed three stages of weight-loss, whereas the DTG curves indicated the presence of a series of thermal peaks associated with the TG curves. The first stage was found to correspond to the thermal dehydration of coordinated water molecules in the first stage. The second stage was corresponding to the formation of intermediate complexes resulted from the decomposition of the dehydrated complexes, whereas the third stage represented to the degradation of the formed intermediates to give metal oxides as final products. These results may be considered as indirect evidence to support our suggested conductance mechanism of the cited work. The thermal decomposition of As (III)-alginate complex stills in progress in our laboratory.

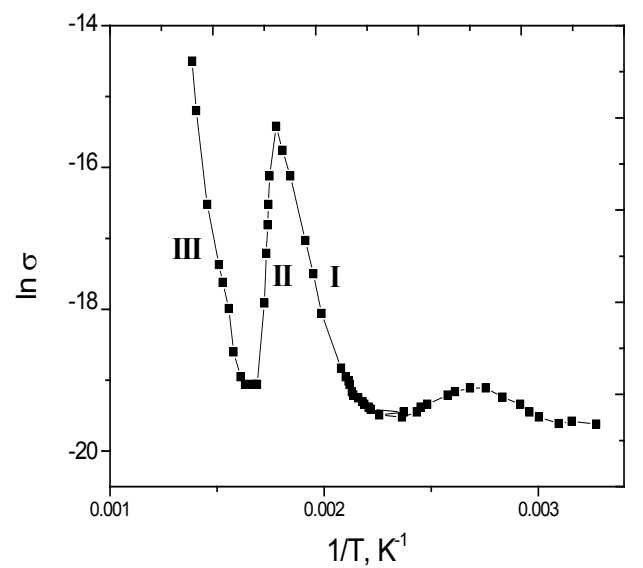

Figure 2: A typical plot of In o against $1 / T$ of coordination biopolymeric arsenic (III)-alginate complex. 
Citation: Zaafarany IA (2014) Temperature-Dependence of Electrical Conductivity for Some Natural Coordination Polymeric Biomaterials Especially Some Cross-Linked Trivalent Metal-Alginate Complexes with Correlation between the Coordination Geometry and Complex Stability. J Adv Chem Eng 4: 111. doi: 10.4172/2090-4568.1000111

Page 4 of 6

It was reported that the interdiffused metal ions chelate the functional groups of alginate macromolecular chains through two geometrical structures [34]. The first type corresponds to an intramolecular association in which the functional groups involved in chelation belong to the same chain. Hence, the plane involving the chelated metal ions is parallel with the plane of alginate macromolecular chains. This configuration may be called a planar geometry. The second structure corresponds to the intermolecular association in which the plane containing the metal ions is perpendicular to the plane of alginate chains. Here, the involved functional groups are related to different chains and the configuration obtained is termed a non-planar geometry. The type and nature of geometry depends on both valency and coordination number of the chelated metal ion in the complex.

Divalent [26] and hexavalent [27] metal cations are known to be of octahedral six coordination geometry in their complexes and, hence, tend to chelate the functional groups via either inter- or intramolecular association in order to attain the octahedral geometry in their complexes as shown in Schemes I and II. The priority of these two geometrical structures depends on the stability of the complexes formed. Again, trivalent- and tetravalent-metal ions are restricted to cross-link the functional groups of alginate macromolecule via only intermolecular association in their alginate complexes for geometrical reasons (Scheme III). The preferability of intermolecular association mechanism for chelation in case of the latter metal- alginate complexes can be explained by their tendency to decrease the bond stretching resulting from metal-oxygen bond elongation occurs in case of intramolecular association [34].

In view of the above interpretation, the geometrical structure in those metal alginate complexes can be achieved through chelation of the interdiffused metal ions with the carboxylate groups as principal units in addition to the hydroxyl and/or the water molecules of the amorphous phase. This is to complete the coordination number of the chelated metal ion and reach with the complex to a more stable geometry.

It is well known that the charge carriers drift their velocity to maximum in case of intermolecular association complexes owing to the presence of channels around the planes containing the metal ions rather than in case of intramolecular association [34-36]. Hence, the electrical conductivity is expected to be higher in non-planar than that in planar geometries [34]. As is shown in Table 2, the value of electrical conductivity lies in the range of semiconductors for monovalent, trivalent and tetravalent metal-alginate complexes indicating that the chelation in these metal-alginate complexes should be of intermolecular association geometry which may confirm our suggestion (Scheme III).

On the other hand, the values of the electrical conductivities for uranium (VI)- and silver(I)-alginate complexes listed in Table 2 may indicate that the uranyl ion prefers to coordinate with the functional group of alginate macromolecule by intramolecular association mechanism (Scheme IV), whereas silver(I) prefers to chelate via intermolecular association ones in its alginate complex (Scheme I) in a good accord with that reported earlier [12,34].

Generally, the conductance in polymeric compounds occurs via two conduction mechanisms named ionic and electronic conduction, respectively, depending on the nature of the charge carriers existing within the network of the macromolecular chains [6]. The value of activation energy may be considered as indirect evidence for the conduction mechanism. When the value of the activation energy, $E_{a}$, is larger than unity, it means that the cationic conduction mechanism tends to be the more prominent one, whereas that value is usually lower than unity in case of electronic conduction mechanism. The activation

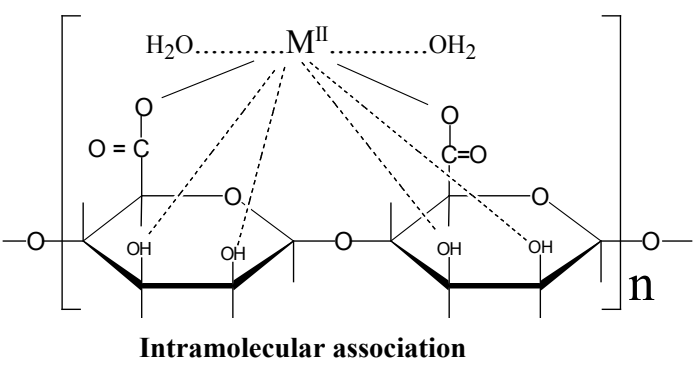

Scheme I: Chelation in Divalent Metal Ion Complexeas

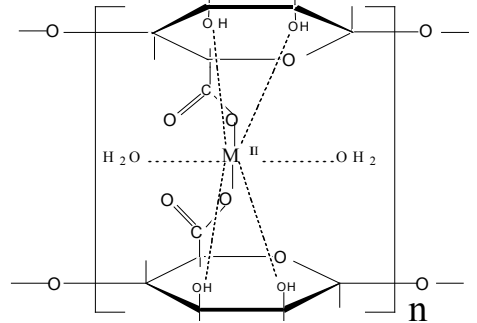

termolecular association
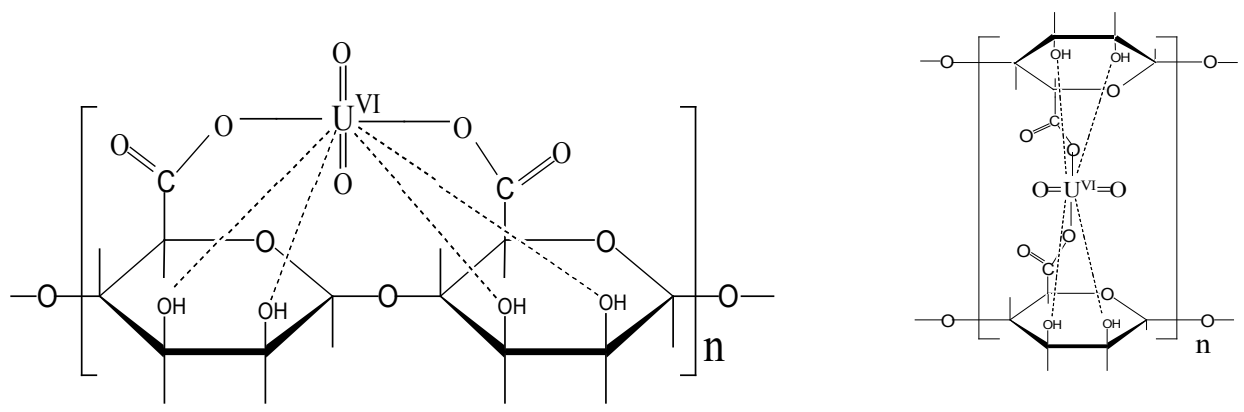

(Intramolecular association)

(Intermolecular association)

Scheme II: Chelation in Uranyl Ion Complex 


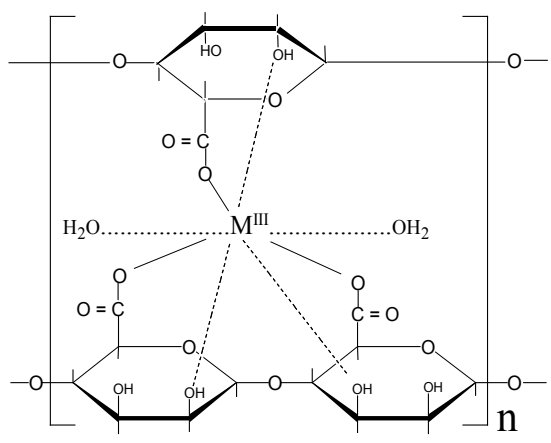

Intermolecular Association

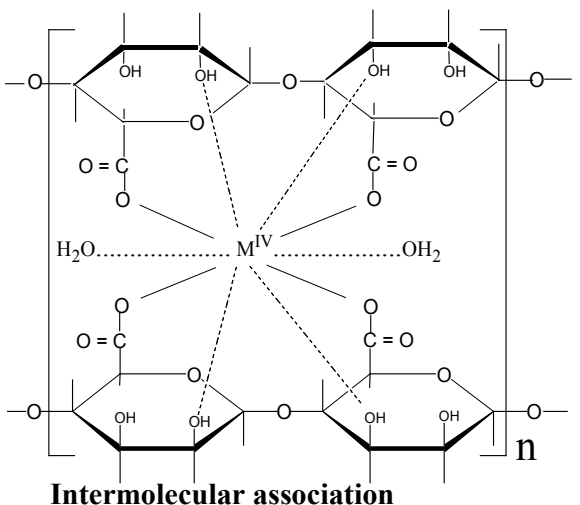

Intermolecular association

Scheme III: Chelation in Trivalent Metal Ion Complexes

\begin{tabular}{|c|c|c|c|c|}
\hline Complex & $\boldsymbol{E}_{a}$ (Initial stage) & $\boldsymbol{E}_{a}($ Final stage $)$ & o' $\left(\Omega^{-1} \mathrm{~cm}^{-1}\right)$ & Reference \\
\hline Ag'-alginate & 0.21 & 3.15 & $1.8 \times 10^{-8}$ & 12 \\
\hline As ${ }^{\prime \prime \prime}$-alginate & 0.13 & 1.64 & $2.6 \times 10^{-9}$ & This work \\
\hline La'll-alginate & - & 1.47 & $1.3 \times 10^{-9}$ & 14 \\
\hline Ce $e^{\text {III-alginate }}$ & - & 1.19 & $1.0 \times 10^{-9}$ & 14 \\
\hline Cr'll-alginate & 0.16 & 2.74 & $10.0 \times 10^{-9}$ & 15 \\
\hline Fe'll-alginate & 022 & 1.41 & $2.0 \times 10^{-9}$ & 15 \\
\hline $\mathrm{Sn}^{\mathrm{IV}}$-alginate & 0.33 & 0.98 & $1.1 \times 10^{-9}$ & 16 \\
\hline $\mathrm{Zr}^{\mathrm{IV}}$-alginate & 0.36 & 0.85 & $1.2 \times 10^{-9}$ & 16 \\
\hline$S e^{I V}$-alginate & 0.25 & 1.17 & $6.0 \times 10^{-9}$ & 17 \\
\hline $\mathrm{Th}^{\mathrm{IV}}$-alginate & 0.86 & 1.20 & $2.01 \times 10^{-9}$ & 18 \\
\hline $\mathrm{U}^{\mathrm{VI}}$-alginate & 0.37 & 0.83 & $1.7 \times 10^{-12}$ & 19 \\
\hline $\mathrm{Ce}^{\mathrm{IV}}$-alginate & 0.28 & 0.84 & $1.04 \times 10^{-9}$ & 37 \\
\hline
\end{tabular}

Table 2: The activation energies in $\mathrm{eV}$ and the electrical conductivity in $\Omega^{-1} \mathrm{~cm}^{-1}(290 \mathrm{~K})$ for some cross linked Metal-Alginate complexes.

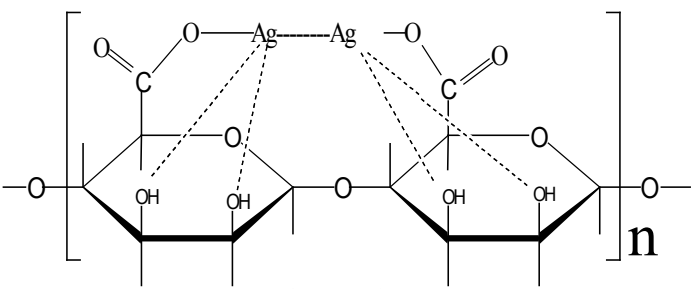

Intramolecular association

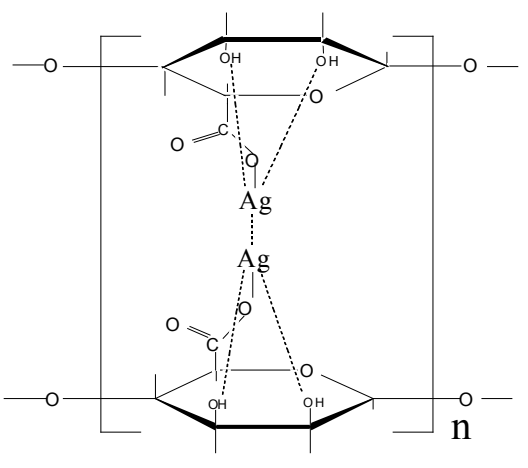

Intermolecular association

Scheme IV: Chelation in Silver (I) Ion Complex

energies in these cross-linked metal alginates can be evaluated from the slopes of $\ln$ o $-1 /$ T plots using the Arrhenius equation

$$
\sigma=\sigma^{\circ} \exp \left(-E_{a} / \mathrm{RT}\right)
$$

where $\sigma^{\circ}$ is the electrical conductivity, $\sigma^{\circ}$ is a constant and $E_{a}$ is the activation energy of the charge carriers. These values were calculated by using the least-squares method and are summarized along with that of other cross-linked metal alginate complexes in Table 2. The calculated values of $\mathrm{E}_{\mathrm{a}}$ were found to be $0.13 \pm 0.005$ and $1.64 \pm 0.04$ for the initial and final stages, respectively. The lower values of activation energy $<1.0$ $\mathrm{eV}$ observed for studied tetravalent metal alginate complexes of the present work may reflect the prominent of the electronic conduction mechanism. The formation of free-radicals through the treatment of the studied complexes can support this suggestion.

\section{Conclusion}

The electrical conductivity of coordination biopolymeric As(III)alginate complex has been measured as a function of temperature. The Arrhenius plot of ln o against $1 / \mathrm{T}$ showed a complicated behavior. The magnitude of the or values indicated that the electrical conductivity of such complex lies in the range of semiconductors. It was found that the geometrical configuration between the chelated metal ion and the functional carboxylate and hydroxyl groups in the determining factor 
Citation: Zaafarany IA (2014) Temperature-Dependence of Electrical Conductivity for Some Natural Coordination Polymeric Biomaterials Especially Some Cross-Linked Trivalent Metal-Alginate Complexes with Correlation between the Coordination Geometry and Complex Stability. J Adv Chem Eng 4: 111. doi: 10.4172/2090-4568.1000111

for the electrical conductivity behavior and play an important role in the stability of the formed complex.

\section{References}

1. Chanda SK, Hirst EL, Percival EG, Ross AG (1952) Structure of alginic acid. J Chem Soc 1833-1837.

2. Nandapure BI, Kondawar SB, Salunkhe MY, Nandapure AI (2013) Magnetic and transport properties of conducting polyaniline / nickel oxide nanocomposites. Adv Mat Lett 4: 134-140.

3. Nhivekar GS, Mudholkar RR (2011) Microcontroller based IR remote contro signal decoder for home application. Adv Appl Sci Res 2: 410.

4. Jing X, Wang Y, Zhang B, (2005) Electrical conductivity and electromagnetic interference shielding of polyaniline/polyacrylate composite coatings. J App Polym Sci 98: 2149-2156.

5. Mead CA (1961) Operation of tunnel-emission devices. J Appl Phys 32: 646.

6. Sulaneck WR, Clark DT, Samuelson EJ (1990) Science and Application of Conducting Polymers. IOP Publishing Ltd., UK, USA.

7. El-Dossouky M, Hegazy EA, Dossuki AM, El-Sawy NM (1986) Electrica conductivity of anionic graft copolymers obtained by radiation grafting of 4-vinylpyridine onto poly(vinyl chloride). Radiat Phys Chem 27: 443-446.

8. Abdel-Wahab SA, Ahmed MA, Radwan FA, Hassan RM, El-Refae AM, et al. (1997) Relative permittivity and electrical conductivity of some divalent metal alginate complexes. Mater Lett 20:183-188.

9. Ahmed MA, Radwan FA, El-Refae AM, Abdel-Wahab SA, Hassan RM (1997) Temperature and frequency dependence of the electrical properties of metal alginate complexes. Ind J Phys 71: 39.

10. Hassan RM, Makhlouf M Th, Summan AM, Awad A (1989) Influence of frequency on specific conductance of polyelectrolyte gels with special correlation between strength of chelation and stability of divalent metal alginate ionotropic gels. Eur Polym J 25: 993-996.

11. Hassan RM (1989) Influence of frequency on electrical properties of acid and trivalent metal alginate ionotropic gels. A correlation between strength of chelation and stability of polye1ectrolyre gels. High Perform Polym 1: 275-284.

12. Hassan RM (1991) Alginate polyelectrolyte ionotropic gels. VII. Physicochemica studies on silver(I) alginate complex with special correlation to the electrical properties and geometrical structure. Coll Surf 60: 203-212.

13. Khairou KS, Hassan RM (2002) Temperature-dependence of electrica conductivity for cross-linked mono- and divalent metal alginate complexes. High Perform Polym 14: 93-99.

14. Zaafarany IA, Khairou KS, Hassan RM (2010) Physicochemical studies on some cross-linked trivalent metal-alginate complexes especially the electrical conductivity and chemical equilibrium related to the coordination geometry. High Perform Polym 22:69-81.

15. Hassan RM, El-Shatoury SA, Mahfouz RM, Osman MA, El-Korashy A, et al. (1995) Alginate polyelectrolyte ionotropic gels. VIII. Electrical properties of di- and trivalent metal alginate complexes specially iron(III) and chromium(III) alginate resins. Bull Fac Sci Assiut Univ Egypt 24 : 141.

16. Hassan RM, Zaafarany IA, Gobouri AA (2013) Temperature-dependence of electrical conductivity of some natural coordination polymeric biomaterials especially cross-linked tetravalent metal-alginate complexes with correlation between the coordination geometry and complex stability. Advan Biosen Bioelectro 2: 16.

17. Zaafarany IA, Khairou KS, Hassan RM, Ikeda Y (2009) Physicochemical studies on cross-linked thorium (IV)-alginate complex especially the electrical conductivity and chemical equilibrium related to the coordination geometry. Arabian J Chem 2: 1-10.

18. Ishaq IA, Khairou KS, Hassan RM (2009) Physicochemical studies on some natural polymeric complexes of quadrivalent metal cations. Electrical conductivity and chemical equilibrium of cross-linked selenium (IV)-alginate complex with correlation between the complex stability and geometrical structure. J Saudi Chem 13: 49-60.

19. Hassan RM, Ikeda Y, Tomiyasu H (1993) Alginate polyelectrolyte ionotropic gels. Part XV. Physicochemical properties of uranyl alginate complex especially the chemical equilibrium and electrical conductivity related to the coordination geometry. J Mater Sci 28: 5143-5147.

20. Awad A, El-Cheikh F (1981) Electrical resistance and anisotropic properties of some metal alginate gels. J Coll Interf 80: 107-110.

21. Hirst E, Rees DA (1965) The structure of alginic acid. Part V. Isolation and unambiguous characterization of some hydrolysis products of the methylated polysaccharide. J Chem Soc 1182-1187.

22. Rees DA, Scott WE (1971) Polysaccharide conformation. Part VI. Compute model-building for linear and branched pyranoglycans. Correlations with biological function. Preliminary assessment of inter-residue forces in aqueous solution. Further interpretation of optical rotation in terms of chain conformation. J Chem Soc B 469-479.

3. Schweiger RG (1962) Acetylation of alginic acid II. Reaction of algin acetates with calcium and other divalent ions. J Org Chem 27: 1786-1789.

24. Hellferich H (1962) lon Exchange, McGraw-Hill, New York.

25. Bellamy LJ (1966) The Infrared Spectra of Complex Molecules. (2 ${ }^{\text {nd }}$ edn), Chapman and Hall, London.

26. Cozzi D, Desider PG, Leppri L, Cinatelli G (1968) Alginic acid, a new thin layer material. J Chromatogr 35: 369-404.

27. Cotton AF, Wilkinson G (1972) Advanced Inorganic Chemistry. (3 $3^{\text {rd }}$ edn), John Wiley, New York.

28. Hassan RM (1992) A review on oxidation of uranium (IV) by polyvalent meta ions. A linear free-energy correlation. J Coord Chem Rev 27: 255-266.

29. Neuberger CS, Helz GR (2006) Thermodynamic assessment and chemica interpretations. Appl Geochem 20: 1218-1226.

30. Said AA, Abd El-Wahab MMM, Hassan RM (1994) Thermal and electrical studies on some metal alginate compounds. Thermochem Acta 233: 13-24.

31. Zaafarany IA, Khairou KS, Terkistani FA, Iqbal S, Khairy M, Hassan RM, et al (2012) Kinetics and mechanisms of non-isothermal decomposition of $\mathrm{Ca}$ (II)-, $\mathrm{Sr}$ (II)- and $\mathrm{Ba}$ (II)- cross-linked divalent metal-alginate complexes. Int J Chem 4: 7-14;Said AA, Hassan RM (1993) Thermal decomposition of some divalent metal alginate gel compounds. Polym Degrad Stab 39: 393-397.

32. Zaafarany IA (2010) Non-isothermal decomposition of $\mathrm{Al}, \mathrm{Cr}$ and $\mathrm{Fe}$ crosslinked trivalent metal-alginate complexes. JKAU 22: 193-202.

33. Terkistani FA, Hassan RM (2012) Kinetics and mechanisms of non-isothermal decomposition of some cross-linked metal-alginate complexes especially trivalent-metal-alginate complexes. Orien J Chem 28: 913-920.

34. Hassan RM (1993) Alginate polyelectrolyte ionotropic gels. XIII. Geometrica aspects for chelation in metal alginate complexes related to their physicochemical properties. Polm Inter 31: 81-86.

35. Thomas DP, Randal TC, Ralph M (2006) Molecular models of alginic acid: Interactions with calcium ions and calcite surfaces. Geochimica et Cosmochimica Acta 70: 3508-3532.

36. Braccini I, Grasso RP, Perez S (1999) Conformational and configurationa features of acidic polysaccharides and their interactions with calcium ions: molecular modeling investigation. Carbohyd Res 317: 119-130. 